\title{
SOBRE LA CONSTRUCCIÓN IDENTITARIA ${ }^{1}$
}

\author{
ON THE CONSTRUCTION OF IDENTITY
}

\section{MARÍA ISABEL TOLEDO JOFRÉ ${ }^{2}$}

\section{RESUMEN}

El artículo presenta una conceptualización de la identidad desde una perspectiva constructivista. Explicita una noción de sujeto, define lo que es identidad como un proceso en permanente construcción y lo diferencia de la noción de identidad como pertenencia. Da cuenta de como se construye la identidad, del rol del contexto y de los Otros en dicha construcción. Refiere al rol de la reflexividad en la construcción identitaria y la corporeidad.

Palabras clave: Sujeto, subjetividad, identidad.

\section{ABSTRACT}

The article presents the conceptualization of identity from a constructivist perspective. It specifies a notion of subject, defines identity as being in constant construction and differentiates it from the notion of identity as a belonging. It informs on how identity is built and the importance of context and Others in this process. It refers to reflexivity during the construction of identity and corporeality.

Keywords: Subject, subjectivity, identity.

Recibido: 18.10.11. Aceptado: 07.01.12.

${ }^{1}$ Este artículo fue elaborado en el marco del proyecto FONDECYT No 1090692, "Ser docente en Chile: tensiones históricas y perspectivas a través del enfoque biográfico y etnográfico (19232011), a realizarse entre los años 2009 a 2012.

2 Profesora Titular Facultad de Psicología, Universidad Diego Portales. Santiago, Chile. E-mail: maria.toledo@udp.cl 
$\mathrm{D}$ URANTE LAS ÚLTIMAS DÉCADAS nuevas conceptualizaciones sobre el sujeto se han sumado al debate de las ciencias sociales. A saber, la noción de 'sujeto instrumental' refiere a la acción del sujeto que es guiada por una racionalidad utilitaria e instrumental. De ahí que, desligado de limitaciones, de normas sociales que lo restrinjan, el sujeto puede liberarse al juego de la competencia, al culto de la realización. La noción del 'sujeto narcisista', construida desde el pensamiento postmoderno, concibe al individuo desvinculado de los discursos colectivos emancipadores. Se trata de un sujeto escéptico en cuanto a los roles que debería asumir, lúdico, frívolo, indiferente a los otros. Aquí se manifiesta la idea del agotamiento de las lógicas de acción integrativa y de las acciones colectivas, de donde aparece el individuo consumidor en una sociedad de masas y de medias, que escapa a los códigos de la modernidad, a través de la estetización de su existencia. La autorrealización y la autonomía animan la acción de este sujeto. También se ha hecho referencia a la noción de 'sujeto movimiento social'. Bajo esta concepción el sujeto no existe como categoría independiente, se ubica en la categoría movimiento social, pues éste encarna a los diferentes sujetos históricos. Finalmente, se postula la noción de 'sujeto del sufrimiento social'. Aquí la subjetividad porta las marcas de la dominación social. El sujeto está sometido a la dependencia y al control social, es víctima de la estructura social (Franssen, 1997).

Sin embargo, aún falta considerar aquellas producciones que han dado lugar a la sociología del sujeto: el trabajo intelectual que pone como centro la noción de experiencia y la capacidad reflexiva del sujeto. Aquí, lo que define al sujeto es su capacidad de volver al pasado para interpretar y reinterpretar sus experiencias. De ahí que el foco se sitúa en las relaciones entre el sí mismo (el sujeto) y los Otros, en las competencias del sujeto para estructurar sus relaciones sociales y, al mismo tiempo, (re)producir el sistema social (Franssen, 1997). Bajo esta perspectiva aparece la noción de subjetivación como eje central de la constitución del sujeto, la que se refiere al trabajo sobre el sí mismo, al ser reflexivo. Entonces, el sujeto es una resultante de la puesta en juego de las relaciones sociales. El sujeto no es un espejo del sistema social. El sujeto tiene la capacidad de autodefinición: está limitado por las relaciones sociales que lo constituyen, pero también tiene una capacidad de respuesta, de creación, de resistencia. El sujeto actualiza su capacidad de administrar las tensiones entre lo que los Otros esperan de él y lo que él espera de sí mismo (Bajoit, 1997). 
Esta noción de sujeto adquiere sentido cuando el sujeto se concibe en el mundo del lenguaje, dado que "... toda toma de la palabra es constitutiva del advenimiento del sujeto" (De Villers, 1996: 114). Pero, el sujeto del habla es, al mismo tiempo, un sujeto histórico. Es producto de la historia, es heredero de las condiciones de existencia de sus ancestros. Entonces, la historia permite comprender cómo cada individuo ha sido llamado a ocupar tal o cual posición social (De Gaulejac, 1991), desde la cual construye su entorno y se construye a sí mismo. El sujeto se construye en interacción dialéctica con el entorno donde tiene lugar su existencia. Entonces, sus condiciones de existencia son producto de las experiencias acumuladas por los grupos a través de la historia (Berger \& Luckman, 1989 en Aznar, 1992).

El sujeto interpreta sus condiciones de existencia y, en ese acto, se va construyendo en interacción permanente con el mundo en que habita. El sujeto crea significados sobre su entorno y se lo apropia, lo transforma o lo hace perdurar en el tiempo.

El sujeto no se construye de una vez y para siempre. El sujeto está en permanente interacción con el entorno en el cual existe. Entonces, mediado por el lenguaje, el sujeto aprehende la realidad, que es el producto de la actividad humana objetivada, y produce la realidad, como resultado de su permanente actividad (Aznar, 1992). En esta constante construcción, el individuo es producido por la historia. Su identidad se construye, por una parte, a partir de los acontecimientos personales que experiencia y que forman la trama de su biografía. Por otra parte, su identidad se nutre de los elementos comunes a su familia y a los colectivos a los cuales pertenece. Así se posiciona como un ser sociohistórico. Sin embargo, si bien la historia actúa sobre el sujeto, no lo determina, porque el sujeto es un ser activo que puede modificar sus prácticas y las estructuras en las cuales se encuentra inserto. Entre el sujeto y las estructuras existe un margen de libertad sobre el cual puede actuar para construir su propia historia. Sin embargo, para construir su propia historia el sujeto debe tomar conciencia de la forma en que la estructura social y la historia colectiva han actuado sobre sus opciones personales. De esta toma de conciencia podrá desarrollar la capacidad de construir historia.

El sujeto también es productor de historias, puesto que cuenta con la capacidad de construir narraciones. Incluso, puede re-significar su trayectoria (De Gaulejac, 1991), no con el propósito de controlar el curso de la historia sino de otorgarle su propio sentido. Entonces, tiene la capacidad para retornar reflexivamente sobre su pasado, proyectarse en el futuro y transformarse en sujeto histórico (Gallez, 1996). Por ello, el sujeto es porta- 
dor de historicidad: de la capacidad de intervenir sobre su propia historia. Él es el producto de una historia, de la cual él busca llegar a ser sujeto.

De este modo, bien se podría afirmar que una vida es una trayectoria en el cual el sujeto se apropia de las relaciones sociales, las interpreta, las re-interpreta y establece otras nuevas. Por ello, cada sujeto es siempre una síntesis de la historia social del colectivo al cual pertenece y, al mismo tiempo, sus prácticas resultan ser una síntesis de la estructura social en la cual se encuentra inserto. Por tanto, el sujeto asume un rol activo en su relación con la historia y la estructura social. "Lejos de reflejar lo social, el individuo lo apropia, lo mediatiza, lo filtra y lo retraduce, proyectándolo en otra dimensión, que corresponde a la de la subjetividad" (Ferrarotti, 1983: 51). El sujeto re-inventa a cada instante lo social y a través de sus prácticas singulariza la universalidad de la estructura social e individualiza la historia colectiva (Ferrarotti, 1983).

En esta construcción a nunca acabar, el sujeto se define a sí mismo como incompleto, por tanto, busca la finitud. Rechaza el mundo tal cual es y actúa para transformarlo, para realizarse como sujeto. Así el sujeto construye su identidad.

\section{SOBRE LA IDENTIDAD}

La identidad se construye en la relación del sujeto con su entorno y con los otros. Esta afirmación sólo evidencia que la identidad refiere a la construcción del sujeto en lo social. Por tanto, la identidad no apunta a la esencia del ser. La identidad no es algo dado, no es fija. No es la suma de características sociales, psicológicas y/o culturales. La identidad no viene dada desde fuera. Los Otros y el entorno son vitales para su construcción. La identidad es una construcción permanente.

La identidad "es el yo entendido reflexivamente por la persona en función de su biografía... supone continuidad en el tiempo y el espacio: pero la identidad del yo, es esa continuidad interpretada reflejamente por el agente" (Giddens, 1997: 72). Sin embargo, no se trata de un acto reflejo sino de la construcción de un concepto de persona, cuyo contenido varía según la cultura (Giddens, 1997). Sin embargo, la identidad no es la misma para todos los miembros de un colectivo (Mucchielli, 2002). La identidad es individual, pero también colectiva. Se construye en la interacción. Por lo tanto, la identidad no es fija. No se corresponde con la respuesta a la pregunta ¿quién soy? Esta pregunta no tiene sentido desde la perspectiva comprehensiva. Esta pregunta conduce al esencialismo, a una cosificación del 
sentido de una vida, a la fijación y, por tanto, a la negación de la identidad. Si la identidad es una construcción, la pregunta ¿quién soy? se transforma en ¿quién estoy siendo? en un momento y contexto particular de la existencia. Entonces, resulta más apropiado hablar de construcción identitaria.

Referirse a la construcción identitaria implica incorporar la dimensión de trayectoria. Una continuidad que permite afirmar que alguien a través del tiempo mantiene una igualdad consigo mismo y al mismo tiempo es diferente a otro. Además, el sujeto tiene conciencia sobre su propia existencia. Sabe que es un mismo sujeto a través del tiempo y que mantiene una coherencia a pesar de los cambios que él mismo realiza, los cambios del contexto y de los Otros que lo rodean (De la Torre, 2001). Sin embargo, la identidad no es una categoría empírica. Ella emerge como respuesta a una acción analítica. Es un constructo que da cuenta de una manera de existir en el mundo y de la conciencia de esa existencia. La identidad se refleja en lo que está siendo el sujeto, es decir, la resultante de lo que ha sido y de lo que desea ser. Por ello, más que definirla, se adjetiva, se le connota y denota. La identidad corresponde al resultado del proceso de apropiación simbólica del conjunto de experiencias que el sujeto encuentra durante su trayectoria vital. Este proceso es vivenciado por el sujeto en tanto actor singular de una situación social determinada.

La identidad es una construcción de la cual el sujeto extrae permanencia y singularidad. La permanencia refiere a lo que él es, siendo siempre un 'ser idéntico a sí mismo' y la singularidad le asegura ser único y no confundirse con otro. Entonces, la identidad está en el corazón de la individualidad. Pero, la identidad siempre se construye en relación con un entorno, se trata de una manera de ser y de situarse en un lugar y de relacionarse con los otros sujetos y los colectivos. Por ello, la identidad constituye una manera de existir en el medio social y define la manera en que el sujeto interpreta la realidad y sus acciones (Chauchat, 1999).

Según Serrano (1997), hay que remarcar que la construcción identitaria corresponde a un proceso continuo y progresivo de construcción en la interacción, inestable y jamás acabado. En este proceso el sujeto tiene un rol activo: construye sentidos y se apropia de las experiencias. Asumiendo su rol activo, podrá devenir constructor de su identidad, podrá definir el curso de su historia y de la historia del colectivo al cual pertenece (Gagnon, 1980), podrá asumir su historicidad.

Sin embargo, la identidad no es únicamente el resultado de la interacción social. Ella es “... también una realidad subjetiva” (Burkitt, 1991 en Veredas, 1999: 115). Es un proceso de construcción de sentido, el más importante y permanente que desarrolla todo sujeto. 
La identidad es producto de una historia, en tanto requiere del desarrollo de una trayectoria. Una trayectoria corresponde a la serie de posiciones que en un espacio en incesante transformación va ocupando un sujeto o un grupo en forma sucesiva (Bourdieu, 1994). Por eso, la identidad puede comprenderse como el transitar por marcos institucionales durante el periodo de existencia del sujeto. De ahí que todo sujeto “... vive una biografía reflejamente organizada en función de los flujos de la información social y psicológica acerca de los posibles modos de vida" (Giddens, 1997: 26).

Como la trayectoria refiere a un sujeto que se desplaza, la identidad es un producto que emerge en la intersección de los procesos psicológicos y sociales que tienen lugar de los contextos en los cuales está inmerso y bajo el entramado de significaciones compartidas por los colectivos con los que interactúa. Así se integra el sujeto a su cultura (Deschamps, Morales, Páez y Wolchel, 1999). Entonces, en su construcción identitaria, el sujeto muta y transmuta en la dimensión espacio-temporal de su existencia en la medida que vivencia nuevas experiencias. La resultante es un producto singular, una identidad particular.

La identidad emerge en singular como resultante de la intersección de las pertenencias del sujeto a través del tiempo (Serres, 1997). De ahí que la identidad no es igual a la pertenencia.

Sin embargo, algunos autores han concebido la identidad como “... una cualidad o conjunto de cualidades con las que una persona o grupo de personas se ven íntimamente conectados" (Larraín, 2001: 23). Entonces, la identidad refiere a como los individuos se definen a sí mismos en función de ciertas características compartidas, a saber: categorías sociales como etnia, religión, nacionalidad; las posesiones materiales y los Otros, en tanto grupo del cual se es parte (Larraín, 2001).

Entonces, se hace necesario establecer la distinción entre identidad y pertenencia.

Desde la teoría de conjunto, la distinción entre estos dos conceptos resulta evidente. Para indicar la igualdad se utiliza un signo igual (=), mientras que para indicar la pertenencia se emplea una épsilon estilizada $(\varepsilon)$. El primer concepto refiere a términos individuales y el segundo indica que un elemento es parte de un grupo (un conjunto), es decir, comparte algún elemento o propiedad del grupo (Serres, 1997 en De Villers 2002). Entonces, no es lo mismo identidad que pertenencia.

En el mundo social, las pertenencias de los sujetos son múltiples y, salvo excepciones, son cambiantes. Entonces, si una pertenencia se define como identidad, el sujeto quedaría reducido a una sola pertenencia. Por ejemplo, su pertenencia a la nación chilena, cuando el ser chileno es una de las 
tantas pertenencias que comparten todos quienes han nacido en el mismo territorio. Si se hacen colectivas las relaciones de pertenencia y se conciben como relaciones unívocas de identidad, se procede de una manera ajena a la teoría de conjuntos, puesto que se incorporan las relaciones de inclusión y exclusión que no son parte de la teoría de conjunto. Entonces, se está frente a un error lógico (Serres, 1997 en De Villers 2002). De ahí que la identidad no corresponde a una o a múltiples pertenencias. Más aún, cuando se trata del análisis de la identidad del sujeto, es necesario considerar que es el principio de no identidad de sí mismo con sí mismo lo que hace posible que el sujeto se constituya en un agente histórico que se transforma a sí mismo. Por ello, su identidad siempre es cambiante (De Villers, 2002). Así, la identidad es un conjunto de significaciones, fijadas momentáneamente, que se construyen en un contexto sociohistórico material e ideacional, más o menos impreciso, en el entramado de pertenencias. La identidad es, entonces, el sentido otorgado por el actor a su propia condición de sujeto. Obviamente, los contenidos de las significaciones varían según la posición del sujeto en la estructura social y del momento socio-histórico en que se encuentre (Mucchielli, 2002).

Dado que la identidad es un sentido otorgado por el sujeto a su propia experiencia, la identidad no puede ser compartida. Cada actor construye su propia identidad, aunque pueda compartir historias, entornos y experiencias con otros miembros de los colectivos a los cuales pertenece. Entonces, existen tantas identidades como sujetos.

Si la identidad se construye a lo largo de la existencia del sujeto, la identidad conlleva una historia. La historia debe considerar sus dos acepciones: la historia como sucesión de acontecimientos y la historia como el relato de dichos acontecimientos (Veyne, 1984). Además, hay que considerar que la historia no refiere sólo al pasado sino también a la historia que está en construcción, es decir, el futuro (Lebovici, 1979 en De Gaulejac, 1991). Entonces, el concepto de identidad también connota la noción de proyecto: una identidad actúa como tal para quien tiene un proyecto. Por ello, la identidad varía según los actores y las situaciones consideradas. Ella es plural. En tanto que la identidad es un asunto de significaciones construidas por el sujeto, en función de su propia identidad y de sus proyectos, ella es recursiva. La identidad no es estática, ni fija. Está en permanente transformación, ya sea por las mutaciones que operan en el entorno y/o los cambios realizados por el propio sujeto, como por los relatos que el sujeto construye sobre su trayectoria vital. Al mismo tiempo, el sujeto modifica las relaciones que establece con su entorno y con los Otros que forman parte de él. Sin embargo, en la mutación y transmutación de la identidad no sólo 
operan procesos externos. La identidad también es resultante de procesos propios del sujeto de carácter biológico, afectivo y cognitivo que se desarrollan en el marco de procesos relacionales y comunicacionales, históricos y culturales (Mucchielli, 2002).

La identidad no es un constructo empíricamente observable, aunque ella se manifiesta en las prácticas que desarrolla el sujeto y le permite construir un relato particular, un relato sobre su propia existencia. Aunque en ese relato incluye ficción, su narrativa no es del todo ficticia, puesto que dicho relato se construye a partir de las experiencias que el sujeto ha vivenciado en el contexto de su existencia y con los Otros que lo rodean. Se trata de un relato que da cuenta de los acontecimientos que el sujeto ha vivenciado a lo largo de su historia. Por ello, Taylor indica que: “... para tener un sentimiento de quienes somos, debemos poseer una idea de cómo hemos llegado a ser y de adónde vamos" (Giddens, 1997: 74).

Entonces, si la identidad es relato, es intriga, es también una construcción de sentidos, cuyos contenidos varían infinitamente, pero está condicionada por las estructuras sociales y los conglomerados de significaciones que regulan las condiciones de existencia del sujeto. Además, hay “... aún otras diferencias más sutiles y también más importantes. Las biografías reflejas difieren en gran medida, de la misma manera que los relatos (en función, por ejemplo, de la forma y el estilo)" (Giddens, 1997).

Las biografías reflejas dan cuenta de una construcción de sentidos. Sin embargo, esos sentidos se construyen sobre las acciones de los sujetos. Como señala Giddens, "... no somos lo que somos, sino lo que hacemos" (1997: 99). Y para avanzar en la construcción de su sí mismo, en la construcción permanente de la identidad, desde el presente, el sujeto se apropia de su pasado y desde ahí se lanza a sus proyectos futuros.

Dado que la identidad es producto de la interacción del sujeto con su entorno, toda identidad es una identidad situada. Entonces, la construcción identitaria ocurre en un territorio. En ese espacio ya apropiado por otros, el sujeto se constituye en un actor social, en la medida que, tomando en cuenta su pasado, desarrolla acciones con perspectiva de futuro. Las acciones se realizan siempre en relación con los Otros que están presentes (de diferentes maneras) en el mismo territorio. También se debe tener presente que ese territorio define -en parte- las posibilidades de acción del sujeto: las favorece, restringe o condiciona. Sin embargo, siempre el sujeto interpreta sus condiciones de existencia, define el sentido y la estrategia de su acción y vuelve a interpretar, a re-significar el territorio. En ese espacio apropiado y re-apropiado, donde tienen lugar las interacciones que el sujeto establece 
con los Otros y las acciones, se puede asir la identidad situada de un sujeto (Mucchielli, 2002).

El sujeto actúa diversas identidades situadas en forma simultánea. No en el instante preciso, pero sí diferenciadas por un breve tiempo. En el tiempo presente se sitúa en relación a los colectivos a los cuales pertenece (Deschamps et al., 1999). De ahí extrae significaciones momentáneamente compartidas. Y cuando se analiza la identidad a lo largo del tiempo, el sujeto suma la cantidad de identidades situadas que ha desplegado a lo largo de su trayectoria vital (Mucchielli, 2002).

Si la identidad es situada, ella se construye en un territorio donde los Otros juegan el rol principal. Esto significa que la identidad se construye en la alteridad. Entonces, el colectivo permite que el sujeto exista, que construya su identidad. Esto acontece porque los Otros han estado presentes incluso antes del lenguaje, están presentes durante toda la trayectoria vital del sujeto. Ellos están presentes como figuras significativas, como referentes culturales, como mitos, como figuras de poder, etc. (De la Torre, 2001). Es por eso que la identidad se construye en y a través de las pertenencias, en los colectivos por los cuales circula el sujeto. Así, los Otros, próximos o lejanos, volátiles o permanentes, constituyen un campo de producción identitaria que genera aperturas o restringe las posibilidades de acción del sujeto. Los Otros definen las posibilidades de enriquecimiento de su identidad, puesto que, mientras más experiencias se compartan con los Otros, mayor es la expansión de la identidad.

La identidad también es la resultante del conjunto de narraciones que circulan en torno al sujeto. Esto, porque las historias vehiculan los significados compartidos por los colectivos. Además, son esas historias sobre el acontecer de la existencia las que permiten las interacciones entre los sujetos y son producto de ellas mismas. Y es a través de las narrativas que el sujeto expresa su identidad a los Otros. Entonces, las narrativas vehiculan proposiciones de identidad que son interpretadas por otros sujetos que portan su propia identidad. Así, “... cada identidad es, a cada instante, una emergencia de sentidos, resultante de un conjunto de negociaciones circulares de identidades de cada uno. Cada identidad encuentra su fundamento, entonces, en el conjunto de las otras identidades que se expresan al interior del sistema de relaciones" (Mucchielli, 2002: 36). Por lo tanto, en la tarea de construcción de su identidad, el sujeto participa de un sistema de relaciones donde se construye a partir de lo que es y en las interacciones que establece. En ese entramado de relaciones significa y re-significa permanentemente su imagen de sí, porque es en las interacciones socia- 
les donde se engendran las tensiones existenciales que debe administrar. Debe seleccionar los elementos que desea integrar a su identidad y los que desea eliminar, lo que desea transformar. En esta gestión de sí mismo, en las elecciones sucesivas e inacabadas, se construye el sujeto. Pero antes de integrarlos adecúa esos elementos, los evalúa, los acomoda con el fin de salvaguardar la coherencia y la singularidad de su identidad (Bajoit, 1997).

\section{REFLEXIVIDAD}

La construcción identitaria necesita de la reflexividad. “... la identidad del yo no es algo meramente dado como resultado de las continuidades del sistema de acción individual, sino algo que ha de ser creado y mantenido habitualmente en las actividades reflejas del individuo" (Giddens, 1997: 72). Según Bruner, la identidad (el yo) se define en función de los significados que le son otorgados, pero también de las prácticas. “... 'el significado del Yo' se alcanza y pone en funcionamiento" (1991: 115) Por ello, la identidad no puede construirse si no entra en acción esta capacidad de reflexión. La reflexividad es, según Bruner, “... nuestra capacidad de volvernos al pasado y alterar el presente en función de él, o de alterar el pasado en función del presente" (1991: 109). No se trata de cambiar el pasado en sí mismo, de cambiar lo acontecido, si no de darle nuevos significados a las experiencias que han tenido lugar a lo largo de la historia. Y, en relación al presente $\mathrm{y}$ al futuro, la reflexividad permite definir sentidos y acciones a realizar. Entonces, la reflexividad es siempre interpretación y re-interpretación. Se trata de la acción de autorreflexión o desarrollo de la autoconciencia, como lo nomina Gergen (2006). Se trata de una acción interpretativa que opera independientemente de la capacidad cognitiva, la capacidad discursiva y del territorio en que se encuentre el sujeto. Lo que se pone en juego es su capacidad de significar y re-significar del sujeto.

La acción reflexiva sobre el sí mismo permite tomar conciencia de la unicidad del sujeto y evidencia su dimensión histórica. Es decir, cuando el sujeto se piensa a sí mismo, puede identificar lo que se mantiene de él, pero también aquello que desea transformar y lo que requiere construir. Su permanencia y su singularidad se manifiestan. Sin embargo, para que la construcción de la identidad se realice, se requiere que la construcción social del yo sea ella misma considerada una construcción (Gergen, 2006).

La reflexividad aplicada al sí mismo para avanzar en la construcción identitaria requiere del pensamiento crítico que permita discriminar acciones y significaciones; necesita de la creatividad para dar posibilidad a lo 
nuevo; reclama de la imaginación para poder proyectar hacia el futuro y exige de la metacognición para articular sentidos y proyectarlos en el tiempo. Entonces, es la capacidad reflexiva la que posibilita la transformación de los sujetos en constructores de su propia historia, es la que hace posible la historicidad del sujeto. La reflexividad guía las acciones del sujeto en su territorio y con los Otros. Como indica Bruner, “... el Yo, utilizando su capacidad de reflexión y de imaginar alternativas, rehúye o abraza o re-evalúa y reformula lo que la cultura le ofrece" (1991: 110)

Además, hay que considerar que la reflexividad no se limita a lo que se puede asemejar a una descripción del pasado, no trata de construir una representación de la realidad. La reflexividad implica también una intervención, implica una práctica sobre el mundo (Potter, 1998). Por ello, la reflexividad entendida como un retorno sobre la propia experiencia del sujeto (Steier, 1992), deja de manifiesto que la acción reflexiva sobre el sí mismo es siempre una acción transformativa. Es lo que De Villers (1996) nomina efecto sujeto, el efecto de la subjetivación.

La capacidad reflexiva del sujeto aplicada a su propia identidad posibilita la historicidad individual. Lo que significa que el sujeto puede adueñarse de los márgenes de libertad que le permiten las estructuras sociales, para aproximarse más a su propio proyecto de futuro que a las expectativas que los Otros trazan sobre él. Así, el sujeto conducirá su historicidad individual entrelazada con la historicidad colectiva de los múltiples colectivos de los cuales forma parte.

La reflexividad operada por el sujeto afecta al cuerpo y a los procesos psíquicos del sujeto (Giddens, 1997). Por ello, el cuerpo no es un mero depositario o continente de la identidad del sujeto.

\section{CORPOREIDAD}

Identidad del sujeto y corporeidad son dos dimensiones difíciles de separar, ya que "el yo, naturalmente, está corporeizado" (Giddens, 1997: 76).

La corporeidad no es sólo una materialidad que contiene o donde habita un sujeto. Tampoco es sólo una fuente de sensaciones placenteras y de aquellas que no lo son. Como otra dimensión del sujeto, la corporeidad es resultante de la acción simbólica del sujeto y de los Otros sobre él, es producto de múltiples prácticas que se realizan directa o indirectamente sobre ella y en relación a ella y de diversas significaciones que se le atribuyen -ya sea al cuerpo como totalidad y a sus órganos de forma independiente-. La corporeidad es un producto más de la acción cultural. 
Más aún, se puede afirmar que el cuerpo es acción y que la corporeidad tiene particulares implicancias sobre las interacciones que se establecen en la vida cotidiana, puesto que vehicula significaciones y permite desarrollar las acciones que se propone el sujeto. Además, las significaciones tienen origen en el cuerpo y del cuerpo emanan las significaciones que hacen posible la existencia individual y colectiva de los sujetos (Le Breton, 2002).

También se debe considerar que, toda acción que realiza el sujeto -sea individual o colectiva, simple o compleja, cotidiana o excepcional, espontánea o planificada- se hace cuerpo, se corporeiza. También el territorio donde su existencia tiene lugar moldea y modifica la corporeidad del sujeto. Al mismo tiempo, todas sus acciones necesitan de su cuerpo.

Desde la más temprana existencia, desde las primarias exploraciones del niño en el mundo, cuando comienza a tomar conciencia de la existencia de su cuerpo y de sus capacidades, es a partir del cuerpo y con el cuerpo que el sujeto establece su relación con su entorno. A través de los órganos de los sentidos, el sujeto accede a la experiencia de habitar un territorio y a partir de eso construye interpretaciones sobre lo que ahí acontece y los sujetos que lo pueblan (Le Breton, 2002). Con su corporeidad, el sujeto se apropia del mundo, lo significa y lo transforma buscando materializar sus significaciones y luego es el mismo entorno -material y simbólico- el que moldea su cuerpo.

En el cuerpo se manifiesta la identidad del sujeto y al mismo tiempo permite su diferenciación de otros sujetos. Como indica Le Breton: "La existencia es, en primer término, corporal" (2002: 7). Además, como el sujeto se inscribe en la temporalidad, la corporeidad no sólo da cuenta de la identidad del sujeto en cada instante sino que también en ella queda plasmada su trayectoria vital. En el cuerpo se graba la historicidad del sujeto.

La corporeidad juega un rol fundamental en el mantenimiento de coherencia de la identidad. Puesto que, a pesar que el relato sobre sí mismo y la corporeidad cambia material y simbólicamente a través del tiempo, se trata siempre de una misma materialidad.

El cuerpo es una resultante de la acción reflexiva del sujeto e inversamente, la reflexividad del sujeto actúa sobre la corporeidad, pues éste se ve activado y/o sometido por diversos sistemas abstractos, regímenes corporales y estilos de vida que lo moldean desde el exterior.

Si el cuerpo es resultante de un sistema de prácticas mediadas por la reflexividad, el cuerpo integra los significados y prácticas con que se interactúa a cotidiano, las condiciones de vida, los regímenes existentes, los estilos establecidos y los otros posibles de crear. El cuerpo también está inmerso 
y cruzado por las construcciones relacionadas con la sexualidad (Giddens, 1997). Entonces, se torna más preciso hablar de corporeidad, pues ésta incluye más que la materialidad. Así, se puede afirmar que la corporeidad es al mismo tiempo resultante y contiene las marcas de las experiencias que ha vivenciado el sujeto. El cuerpo también es un recurso para marcar y comunicar la identidad y las pertenencias de las identidades situadas del sujeto.

\section{REFERENCIAS}

Aznar, Pilar (1992). "El constructivismo en educación”. En Aznar, Pilar (Ed.). Constructivismo y educación, pp. 13-50. Valencia, España: Tirant to Blanch.

Bajoit, Guy (1997). “Qu'est-ce que le sujet?”. En Bajoit, Guy y Emmanuel Belin (Eds.). Constributions à une sociologie du sujet (pp. 113-130). París, Francia: L'Harmattan.

Berger, Peter y Luckman, Thomas (1989). La construcción social de la realidad. Madrid, España: Amorrortu.

Bruner, Jerome (1991). Actos de significado: más allá de la revolución cognitiva. Madrid, España: Alianza.

Burkitt, Ian (1991). Social selves: theories of the social formation personality. Londres, Inglaterra: Sage Publications.

Chauchat, Hélène (1999). "Du fondement social de l'identité du sujet" (pp. 7-26). En Chauchat, Hélène y Durand-Delvigne, Annick (Eds.), De l'identité du sujet au lien social. Paris, Francia: Presses Universitaires de France.

De Gaulejac, Vincent (1991). La névrose de classe. París, Francia: Hommes et Groupes Éditeurs.

De la Torre, Carolina (2001). Las identidades. Una mirada desde la psicología. La Habana, Cuba: Centro de Investigaciones y Desarrollo de la Cultura Cubana Juan Marinillo.

De Villers, Guy (1996). "L'approche biographique au carrefour de la formation des adultes, de la recherche et de l'intervention. Le récit de vie comme approche de recherche - formations". En Desmarrais, Danielle y Pilon, JeanMarc (Eds.), Pratiques des histoires de vie. Au carrefour de la formation, de la recherche et de l'intervention (pp. 107-134). París, Francia: L'Harmattan.

------ (2002). “El sujeto dividido y el deseo de formación”. Praxis 4, 100-111.

Deschamps, Jean-Claude; Morales, Francisco; Páez, Darío y Wolchel, Stephen (1999). L'identité sociale. La construction de l'individu dans les relations entre groupes. Grenoble, Francia: Presses Universitaires de Grenoble.

Ferrarotti, Franco (1983). Histoires et histoires de vie. La méthode biographique dans les sciences sociales. París, Francia: Librairie des Méridiens.

Franssen, Abraham (1997). "Balises et écueils d'une sociologie du sujet". En Bajoit, Guy y Belin, Emmanuel (Eds.), Constributions à une sociologie du sujet, pp. 17-50. París, Francia: L'Harmattan. 
Gagnon, Nicole (1980). "Données autobiographiques et praxis culturelle", en Cahiers internationaux de sociologie 69, 291-304.

Gallez, Danielle (1996). "A la recherche de nos filiations", Pratiques de formation (Analyses). Les filiations théoriques des histoires de vie en formation,31, 13-21.

Gergen, Kenneth (2006). El yo saturado. Dilemas de identidad en el mundo contemporáneo. Barcelona, España: Editorial Paidós.

Giddens, Anthony (1997). Modernidad e identidad del yo. El yo y la sociedad en la época contemporánea. Barcelona, España: Ediciones Península.

Larraín, Jorge (2001). La identidad chilena. Santiago, Chile: Lom Ediciones.

Le Breton, David (2002). La sociología del cuerpo. Buenos Aires, Argentina: Ediciones Nueva Visión.

Lebovici, Serge (1979). Névrose Infantile et névrose de transfert. $39^{\mathrm{e}}$ Congrès des Psychanalystes de Langue Française. París, Francia: Presses Universitaires de France.

Mucchielli, Alex (2002). L'identite. París, Francia: Presses Universitaires de France.

Potter, Jonathan (1998). La representación de la realidad. Discurso, retórica y construcción social. Barcelona, España: Editorial Paidós.

Serrano, Amparo (1997). "Le sujet comme processus inachevé". En Bajoit, Guy y Belin, Emmanuel (Eds.). Contributions à une sociologie du sujet. pp. 95111. París, Francia: L'Harmattan.

Serres, Michel (1997). “¿Qu'est-ce que l'identité?”. Le Monde de l'Education, de la Culture et de la Formation 214, p. 6.

Steier, Frederick (editor) (1992). Researcho and reflexivity. London, Inglaterra: Sage Publications.

Veredas, Sonia (1999). "Procesos de construcción de identidad entre la población inmigrante". Paper 57, 113-129.

Veyne, Paul (1984).¿Cómo se escribe la historia? Foucault revoluciona la historia. Madrid, España: Alianza Editorial. 